\title{
Sigmoid Volvulus, Intestinal Obstruction and Bowel Perforation; an Unusual Presentation of Pedunculated Fibroid Torsion. Case Report
}

\author{
Emmanuel Mamah ${ }^{*}$, Azubuike Onyebuchi ${ }^{2}$, Chichetaram Otu ${ }^{3}$, Nnaemeka Egbuonu ${ }^{4}$, \\ Parakrama Happawana ${ }^{1}$ \\ ${ }^{1}$ Department of Obstetrics and Gynaecology, Homerton University Hospital NHS Foundation Trust, London, UK \\ ${ }^{2}$ Federal Medical Centre, Umuahia, Nigeria \\ ${ }^{3}$ Government House Clinic, Abakaliki, Nigeria \\ ${ }^{4}$ Queens Hospital, Romford, Essex \\ Email: *johnbosco.mamah.pg78545@unn.edu.ng
}

How to cite this paper: Mamah, E., Onyebuchi, A., Otu, C., Egbuonu, N. and Happawana, P. (2021) Sigmoid Volvulus, Intestinal Obstruction and Bowel Perforation; an Unusual Presentation of Pedunculated Fibroid Torsion. Case Report. Case Reports in Clinical Medicine, 10, 308-313.

https://doi.org/10.4236/crcm.2021.1010039

Received: September 25, 2021

Accepted: October 18, 2021

Published: October 21, 2021

Copyright $\odot 2021$ by author(s) and Scientific Research Publishing Inc. This work is licensed under the Creative Commons Attribution International License (CC BY 4.0).

http://creativecommons.org/licenses/by/4.0/

\begin{abstract}
Pedunculated fibroid torsion presenting as a case of acute abdomen from sigmoid volvulus and large bowel perforation is rare. Without prompt diagnosis and intervention, this could lead to serious morbidity and mortality. Ms FM was a 52-year-old perimenopusal woman who was admitted to the Intensive Therapy Unit (ITU) with worsening symptoms of confirmed Covid-19 infection. On the 10th day of her admission, she developed abdominal distension and tenderness. A pelvic ultrasound scan showed a large pedunculated fibroid measuring $23 \times 15 \times 22 \mathrm{~cm}$. The plan was for conservative management to use pain killers. Following deterioration of her clinical state, an abdominal CT scan was done which confirmed a large uterine fibroid, large bowel distention. CT findings also showed sigmoid volvulus and large bowel perforation. Following a multidisciplinary team assessment, she had an emergency exploratory laparotomy with findings of a large, torted, pedunculated fibroid with adherent sigmoid colon which had become twisted and obstructed. The large bowel segment above the Sigmoid volvulus was grossly distended and there was a gangrenous hepatic flexure with perforation. She had a right hemicolectomy, a de-functioning colostomy and subtotal hysterectomy. Postoperatively, she made very good clinical improvement. Fibroid histology report showed tissue infarction and necrosis which confirmed the torsion. She was discharged home after making good recovery.
\end{abstract}

\section{Keywords}

Case Report, Bowel Perforation, Fibroid Torsion, Pedunculated Fibroid, 
Sigmoid Volvulus

\section{Introduction}

Torsion of a pedunculated leiomyoma associated with Sigmoid volvulus and large bowel perforation is a rare complication [1] [2]. This is considered a surgical emergency due to the risk of ischemic tissue necrosis and acute abdomen. A twisted loop of bowel and its mesentery about a fixed point at the base is known as a volvulus [2] [3]. It may involve the sigmoid colon, cecum, splenic flexure, and transverse colon, in descending order of frequency [1] [2] [3]. The mechanism of volvulus occurrence following fibroid torsion might be related to adhesion of the sigmoid mesentery to the pedunculated uterine fibroid which undergoes torsion and takes with it, the sigmoid colon. This may lead to tissue necrosis, gangrene and bowel perforation.

Clinical diagnosis of fibroid torsion should be considered in women known to have uterine fibroid presenting with acute abdomen and other differential diagnosis should be excluded. Relevant investigation tools including magnetic resonance imaging or abdominopelvic CT scan should be organized to confirm the cause of acute abdomen. Definitive management depends on the individual patient characteristics.

\section{Case Report}

We present the case of Ms FM who presented to our unit with worsening symptoms of Covid-19 infection. Ms FM was a 52 year old perimenopausal woman. She attended the accident and emergency with worsening symptoms of Covid-19 infection and was admitted to the Intensive Therapy Unit (ITU). Following improvement in her clinical condition, she was stepped down from the ITU and transferred to the ward after 7 days of ITU admission. Three days following her transfer to the ward, she complained of nausea, constipation, and abdominal pain; she was given laxatives which led to minimal relief of her symptoms. About forty eight hours later, she developed abdominal distension with tenderness.

\section{Investigations/Diagnosis}

A pelvic ultrasound scan was done which showed a pedunculated fibroid measuring $23 \times 15 \times 22 \mathrm{~cm}$. With worsening symptoms, an abdominal computerised tomography scan (CT) was performed which showed a large fibroid mass projecting from the uterine fundus (blue arrow in Figure 1) and pressing on a dilated large bowel (yellow arrow in Figure 1 and green arrow in Figure 2). The general surgery team reviewed her and advised conservative management. As her symptoms continued to deteriorate, she had a further abdominal CT scan 48 hours later which showed sigmoid volvulus with markedly distended large bowel (green arrow in Figure 2) and evidence of large bowel perforation. 


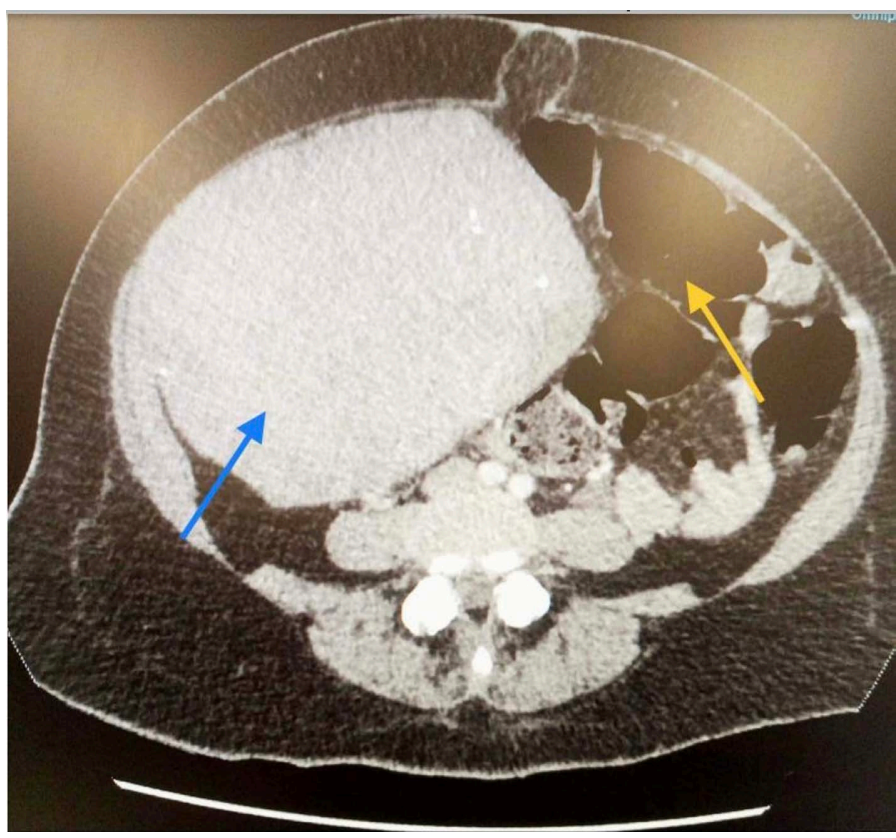

Figure 1. Axial view of computed tomography scan (CT) of abdominal pain. The blue arrow indicates a large uterine fibroid mass pressing on the large bowel which is highlighted by the yellow arrow. The large bowel loop shows evidence of dilatation.

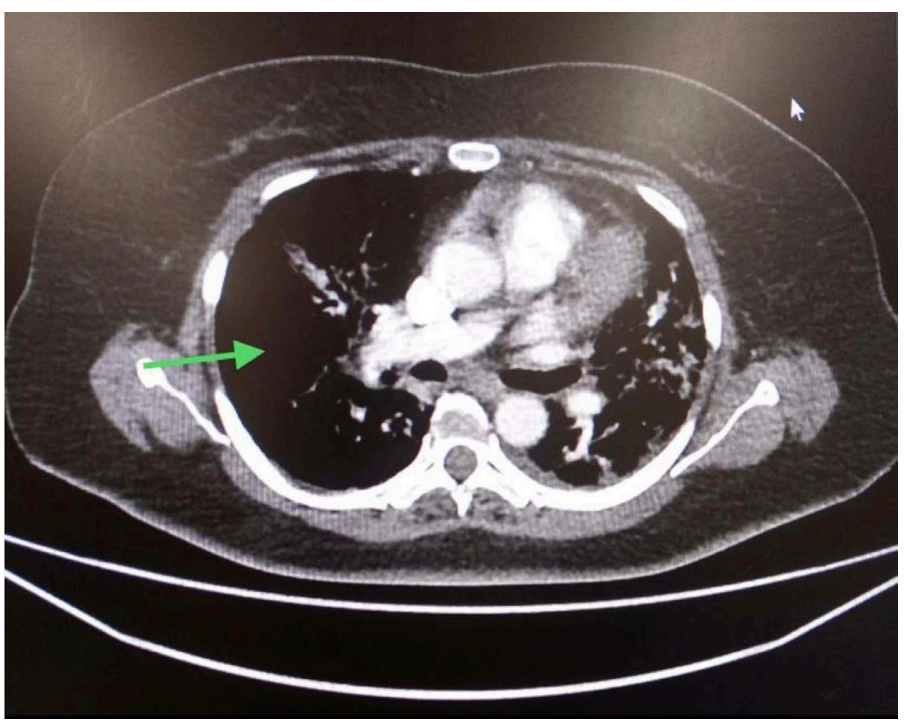

Figure 2. Axial view of computed tomography scan (CT) of the abdomen and pelvis done 48 hours after the initial CT (Figure 1) as a result of worsening symptoms. The green arrow highlights a markedly distended large bowel loop caused by sigmoid volvulus and obstruction as a result of fibroid torsion.

\section{Treatment}

Following a CT-scan finding of possible bowel perforation and with worsening clinical state, she was counselled and consented for an exploratory laparotomy. Intraoperative findings were those of a large, torted, pedunculated fibroid with 
adherent mesentery of the sigmoid colon which had become twisted, causing an obstruction. There was a large bowel distention above the volvulus and there was gangrene and bowel perforation at the hepatic flexure. She had a right hemicolectomy, de-functioning colostomy and subtotal hysterectomy. Postoperative condition remained stable.

\section{Follow-Up and Outcomes}

Postoperative fibroid histology showed fibroid infarction which was a confirmation of tissue necrosis following fibroid torsion. Two weeks following her surgery, she had made very good recovery and was discharged from gynaecological management.

\section{Limitation}

One limitation of this case report is that pictures where not taken during the surgery. This was because the procedure was performed at night and under general anaesthesia. This was not discussed with the patient before she was put to sleep.

\section{Discussion}

Acute abdomen from torsion of pedunculated uterine fibroid is very rare especially when this is associated with sigmoid volvulus [2]. The index patient was unwell with Covid-19 infection which contributed to a diagnostic and surgical challenge. The mechanism of occurrence of volvulus involves twisting of a mobile viscus about a fixed point. In the present case, the mesentery of the sigmoid colon was adherent to the pedunculated fibroid which underwent torsion and with this, there was twisting of the sigmoid colon with subsequent bowel distension, ischaemic necrosis and perforation [2] [3]. Patients with colonic volvulus may present with non-specific symptoms of abdominal pain, constipation, nausea, and vomiting with spontaneous resolution or worsening as was the case with Ms FM [4] [5] [6] [7] [8]. Worsening abdominal pain more often than not indicates possible bowel gangrene and bowel perforation [3].

Making a diagnosis from history and clinical examination findings may not be enough to confirm a diagnosis of fibroid torsion with colonic volvulus. An abdominal CT scan is the imaging modality of choice for the diagnosis of sigmoid volvulus in addition to excluding other potential causes of acute abdomen [1] [2] [3] [4] [5]. As was the case with Ms FM, the initial CT abdomen and pelvic ultrasound scans were unremarkable but subsequent imaging revealed significant bowel distention and gut perforation. Torsion of a uterine leiomyoma could be identified by both CT and pelvic ultrasound (USS) imaging modalities although USS is less sensitive as a screening tool, possibly because its accuracy depends on the the technical knowhow of the individual performing the investigation [9] [10].

When a diagnosis of intestinal volvulus and bowel perforation is confirmed, 
immediate surgical exploration, detorsion and resection of gangrenous bowel segments is recommended to mitigate morbidity and mortality [8] [11]. Following resection, primary anastomosis or colostomy should be undertaken depending on the clinical situation [1] [5] [7] [10]. Sub-total hysterectomy as was done in this case because she had completed her family and hysterectomy would prevent future recurrence. Postoperatively, she made a remarkably good recovery and was discharged with no events. She remained on follow-up and was made abundantly aware of the fact that she retained her cervix and would require cervical smears as long as she remained eligible. This case highlights the complexity of the diagnosis of fibroid torsion complicated by colonic volvulus in a patient with symptomatic Covid-19 infection. The benefits of proceeding with a major surgery should be balanced against the risks and should be discussed with the patient. Detorsion of volvulus and surgical resection should be should be the case where bowel gangrene has occurred.

\section{Conclusion}

This case has shown that sigmoid volvulus from fibroid torsion can present as a life threatening acute abdomen. It is important to consider the possibility of a fibroid torsion in a woman with an abdomino-pelvic mass. Prompt recognition and urgent surgical exploration should be considered sooner rather than later.

\section{Acknowledgements}

We acknowledge all members of the multidisciplinary team that co-managed the patient and ensured a good outcome.

\section{Informed Consent}

Patient gave informed consent for this case/images to be reported for the purpose of advancing scientific knowledge.

\section{Author Contributions}

$\mathrm{EM}, \mathrm{PH}$ and $\mathrm{AO}$ conceptualised the case. Literature review was done by EM, AO, $\mathrm{CO}$ and NE. Initial draft was written by EM and $\mathrm{AO}$ with contributions from $\mathrm{CO}, \mathrm{PH}$ and NE. The final manuscript was read and approved by all the authors.

\section{Data Availability}

The authors declare that data supporting the findings of this study are available within the article.

\section{References}

[1] Arya, V. (1997) Sigmoid Volvulus Caused by Pedunculated Uterine Leiomyoma Leading to Acute Intestinal Obstruction in Young Adult Female. https://www.researchgate.net/publication/284187839

[2] Hilbert, S., et al. (2015) Caecal Volvulus Caused by a Large Uterine Leiomyoma. In- 
ternational Journal of Surgery Case Reports, 10, 97-99.

[3] Mustafa, H., Basak, F. and Alimoglu, O. (2012) Caecal Volvulus. Indian Journal of Surgery, 74, 476-479. https://doi.org/10.1007/s12262-012-0432-9

[4] Madiba, T.E. and Thomson, S.R. (2002) The Management of Caecal Volvulus. Diseases of the Colon \& Rectum, 45, 264-267. https://doi.org/10.1007/s10350-004-6158-4

[5] Consorti, E.T. and Liu, T.H. (2005) Diagnosis and Treatment of Caecal Volvulus. Postgraduate Medical Journal, 81, 772-776.

https://doi.org/10.1136/pgmj.2005.035311

[6] Bajis, R. and Eloundou, G. (2018) Unusual Case of a Torted Mesenteric Fibroid. Case Reports in Obstetrics and Gynecology, 2018, Article ID: 8342127. https://doi.org/10.1155/2018/8342127

[7] Gingold, D. and Murrell, Z. (2012) Management of Colonic Volvulus. Clinics in Colon and Rectal Surgery, 25, 236-244. https://doi.org/10.1055/s-0032-1329535

[8] Montes, H. and Wolf, J. (1999) Caecal Volvulus in Pregnancy. American Journal of Gastroenterology, 94, 2554-2556. https://doi.org/10.1111/j.1572-0241.1999.01394.x

[9] David Le, B.S., Courtney, B.D. and Kathy, B. (2020) Imaging Findings of a Torsed Pedunculated Uterine Leiomyoma: A Case Report. Radiology Case Reports, 15, 144-149. https://doi.org/10.1016/j.radcr.2019.11.001

[10] Roy, C., Bierry, G., El Ghali, S., Buy, X. and Rossini, A. (2005) Acute Torsion of Uterine Leiomyoma: CT Features. Abdominal Radiology, 30, 120-123.

[11] Majeski, J. (2005) Operative Therapy for Caecal Volvulus Combining Resection with Colopexy. The American Journal of Surgery, 189, 211-213.

https://doi.org/10.1016/j.amjsurg.2004.11.004 